\title{
Mechanism and pharmacological specificity of dUTPase-mediated protection from DNA damage and cytotoxicity in human tumor cells
}

Received: 19 August / Accepted: 16 December 1997

\begin{abstract}
Purpose: We have reported previously that the expression of E. coli dUTPase (dutE) can protect HT29 cells from 5-fluorodeoxyuridine (FdUrd)-induced DNA fragmentation and cytotoxicity. In the study reported here, we further characterized the ability of dutE expression in one HT29 clone, dutE7, to alter the effects of treatment with FdUrd and other thymidylate synthase (TS) inhibitors. In addition, we developed two HuTu80 dutE-expressing clones using a pLNCX-dutE retroviral construct and tested their sensitivity to FdUrd-induced DNA fragmentation and cytotoxicity. Methods: Both a dutE retroviral expression system and a dutE antibody were developed to facilitate the generation and screening of dutE-expressing clones. HT29 and HuTu80 clones expressing dutE were tested for drug-induced DNA damage with either alkaline elution or pulsed field gel electrophoresis and drug-induced loss of clonogenicity. Results: Following a 24-h treatment with $100 \mu M$ CB3717 or $500 \mathrm{nM}$ methotrexate (MTX), dutE7 cells were significantly less sensitive to drug-induced loss of clonogenicity than con 3 cells. DutE7 cells were also re-
\end{abstract}

This work was supported by NIH grants P01-CA 42761, R01-CA 56663, R29-CA 53440, NIH Pharmacological Sciences Training Grant 2T32 GM07767 (L.A.P.) and a Predoctoral Fellowship from the University of Michigan Medical School Cancer Research Committee (L.A.P.).

L.A. Parsels · J.D. Parsels · L.M. Wagner

T.L. Loney · J. Maybaum

Department of Pharmacology,

University of Michigan Medical School,

Ann Arbor, MI 48109-0504, USA

E.H. Radany

Department of Radiation Oncology,

University of Michigan Medical School,

Ann Arbor, MI 48109-0504, USA

J. Maybaum ( $\square)$

4701 Upjohn Center for Clinical Pharmacology,

University of Michigan Medical School,

Ann Arbor, MI 48109-0504, USA

e-mail: maybaum@umich.edu sistant to CB3717-induced DNA fragmentation at $24 \mathrm{~h}$. However, following a 48-h treatment with CB3717 or MTX there was no difference in survival between con3 and dutE7 cells, even though DNA damage was still greatly attenuated in the dutE7 cell line. In addition, expression of dutE in two HuTu80 clones, $80 \mathrm{C}$ and $80 \mathrm{~K}$, did not protect these cells from FdUrd-induced DNA damage or cytotoxicity. Conclusions: We conclude that the role of uracil misincorporation and subsequent DNA damage in cytotoxicity induced by TS inhibitors, in HT29 cells, is time dependent, and that cytotoxicity caused by long-term exposure to these drugs is largely independent of resultant DNA damage, in this cell line. The inability of dutE to protect HuTu80 cells from FdUrd further suggests that the significance of uracil misincorporation resulting from TS inhibition varies among cell lines.

Key words dUTPase $\cdot$ Thymidylate synthase DNA damage $\cdot$ Uracil $\cdot$ Resistance

Abbreviations $C d A$ 2-chlorodeoxyadenosine · $D S B$ double strand break $\cdot d u t E$ E. coli dUTPase $F_{\text {released }}$ fraction radioactivity released $\cdot F d U r d 5$ fluorodeoxyuridine $P F G E$ pulse field gel electrophoresis $P B S$ phosphate buffered saline $S S B$ single strand break $\cdot T S$ thymidylate synthase

\section{Introduction}

The fluoropyrimidines, particularly 5 -fluorouracil and 5fluorodeoxyuridine (FdUrd) are the most widely used agents for treating gastrointestinal tumors. One initial biochemical lesion induced by these drugs is inhibition of the enzyme thymidylate synthase (TS) via formation of a covalent ternary complex comprising TS, FdUMP and reduced folate cofactor. TS inhibition has two immediate consequences that are thought to be important in the eventual loss of cellular viability: depletion of dTTP, resulting in DNA synthesis inhibition [13], and an 
accumulation of the enzyme's substrate, dUMP, which is further phosphorylated to dUTP [8]. When dTTP levels are depressed, dUTP is readily incorporated into DNA where it is subsequently excised by the enzyme uracil DNA glycosylase $[8,9,17]$. If no dTTP is available to fill the excised portion of DNA, dUTP will once again be incorporated and excised. This recurring process of uracil misincorporation and misrepair (also called the 'futile repair cycle') is thought to lead eventually to irreversible DNA fragmentation and loss of clonogenic capacity $[9,14,21]$.

The enzyme dUTPase provides the cell with one line of defense against this 'futile repair cycle' $[2,10]$. dUTPase catalyzes the conversion of dUTP to dUMP, inhibiting the accumulation of dUTP associated with TS inhibition. Previous work in our laboratory has shown that excess dUTPase activity is associated with a natural form of resistance to TS inhibitors [4] and has also demonstrated that exogenously expressed E. coli dUTPase (dutE) partially protects HT29 cells from FdUrdinduced cytotoxicity and DNA damage [5].

To better understand the potential therapeutic significance of dUTPase expression in the context of TS inhibition, we expanded our studies to determine the pharmacological specificity of the effects of exogenously expressed dUTPase in HT29 cells, as well as the cell-line specificity of dUTPase protection from the cytotoxic effects of TS inhibition.

\section{Materials and methods}

Cell culture and drug solutions

Con3 and dutE7 are clonal derivatives of the HT29 human carcinoma cell line which express either the neomycin resistance gene or dutE, respectively [5]. Con3, dutE7 and HuTu80 clones (derived from a second human carcinoma cell line, HuTu80) were maintained in McCoy's 5 A medium (Gibco, Grand Island, NY) supplemented with $10 \%$ fetal bovine serum (Intergen, Purchase, NY) and $2 \mathrm{n} M$ L-glutamine (Sigma, St. Louis, MO) at $37^{\circ} \mathrm{C}$ in an atmosphere containing $5 \% \mathrm{CO}_{2}$. GP $+\mathrm{E} 86$ and $\mathrm{GP}+$ envAM 12 cells are ecotropic (E86) and amphotropic (AM12) retroviral producer cell lines grown under similar conditions in DMEM (Gibco, Grand Island, NY) supplemented with $10 \%$ calf serum (Intergen) and $2 \mathrm{n} M$ L-glutamine [18, 19]. FdUrd, 2-chlorodeoxyadenosine (2CdA), and methotrexate (MTX; Sigma) were each dissolved in Hank's buffered saline solution (Gibco) and stored at $4{ }^{\circ} \mathrm{C}$ for up to 2 months. MTX was stored in the dark. CB3717 was dissolved in $10 \mathrm{mM} \mathrm{NaHCO}$ and stored at $-20{ }^{\circ} \mathrm{C}$.

Retrovirus-mediated transduction of cells for expression of dutE

The dutE gene was excised from a previously described pUC.dutE plasmid [5] with EcoRI and ligated into pGEM7zf + (Promega, Madison, Wis.) at the EcoRI multiple cloning site. The sense dUTPase gene was removed from pGEM7.dutE with ClaI and XhoI, gel isolated and cloned into a modified pLNCX retroviral vector between the ClaI and XhoI cloning sites. pLNCX.dutE complemented BW286 bacterial cells at the restrictive temperature [7].

pLNCX.dutE was transfected into ecotropic producer GP + E86 cells using DOTAP transfection reagent (Boerhinger Mannheim). The resultant G418-resistant clones were screened by Western blot analysis for dutE expression and retroviral supernatant from a clone expressing high levels of dutE was used to infect

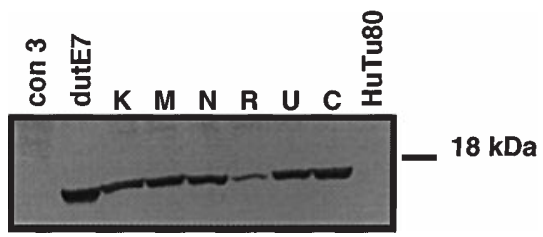

Fig. 1 Western blot analysis of cell extracts from con3, dutE7 and HuTu80 dutE-expressing clones. Cellular proteins were separated by SDS-PAGE and probed with polyclonal anti-dutE antibody. Each lane was loaded with $125 \mu \mathrm{g}$ protein. Lanes $K, M, N, R, U$ and $C$ contain cell extracts from individual HuTu80 dutE-expressing clones

amphotropic producer GP +envAM12 cells in the presence of $8 \mu \mathrm{g} /$ $\mathrm{ml}$ polybrene. Conditioned medium from one AM12 dutE clone was subsequently used to infect HuTu80 cells as described above. Two HuTu 80 clones, $80 \mathrm{C}$ and $80 \mathrm{~K}$, selected by Western blot analysis for expression of high levels of dutE, were further screened for dUTPase activity and FdUrd sensitivity (Fig. 1).

Development of a dutE polyclonal antibody

The dutE gene was cloned into a glutathione S-transferase-conjugating expression vector, pGEXKT at the BamHI cloning site [11] The resultant construct, pGEX.dutE, was used to transform BL21 E. coli, a protease-deficient strain used to generate large amounts of protein [12]. The dutE-glutathione-S-transferase fusion protein (dutE-GST) was purified by affinity chromatography using glutathione agarose [11] and assayed for catalytic activity as described below. Purified dutE-GST was sent to Cocalico Biologicals (Reamstown, $\mathrm{Pa}$.) for use in generating rabbit anti-dutE polyclonal antibody sera.

DutE western blot analysis

Cellular proteins were separated by SDS-PAGE on a $12 \%$ polyacrylamide gel, transferred to a polyvinylidene difluoride membrane (Millipore Immobilon-P) and probed with a 1:5000 dilution of rabbit dutE-antiserum in $1 \%$ milk/20 mM Tris, $500 \mathrm{mM} \mathrm{NaCl}$, $0.1 \%$ Tween-20 (TTBS). After washing with TTBS, the blots were incubated first with a 1:2000 dilution of biotin-conjugated goat antirabbit IgG (Sigma) and then with a 1:2000 dilution ExtrAvidinalkaline phosphatase complex (Sigma), washing again with TTBS between incubations. The dutE band was visualized with nitroblue tetrazolium, 5-bromo-4-chloro-3-indolyl phosphate precipitation (Sigma).

Measurement of dUTPase catalytic activity

Cell extracts were assayed for dUTPase activity as described by measuring the conversion of $\left[5-{ }^{3} \mathrm{H}\right] \mathrm{dUTP}$ to $\left[5-{ }^{3} \mathrm{H}\right] \mathrm{dUMP}$ [4]. Cells were harvested and resuspended at $5 \times 10^{7}$ cells $/ \mathrm{ml}$ extraction buffer $(0.01 M$ Tris $\mathrm{HCl}, \mathrm{pH} 7.5,2 \mathrm{~m} M \beta$-mercaptoethanol, $4 \mathrm{~m} M$ $\mathrm{MgCl}_{2}, 10 \%$ glycerol and $0.2 \mathrm{mM}$ phenylmethylsulfonyl fluoride) prior to freeze-thawing and sonication. Sonicated extracts, adjusted to $0.15 \mathrm{M} \mathrm{KCl}$, were centrifuged at $13000 \mathrm{~g}$ for $10 \mathrm{~min}$ at $4^{\circ} \mathrm{C}$, diluted to $0.2 \mu \mathrm{g}$ protein $/ \mu \mathrm{l}$ extraction buffer, and stored at $-70^{\circ} \mathrm{C}$. For kinetic dUTPase assays, protein extracts were incubated with $0.05 M$ Tris $\mathrm{HCl}, \mathrm{pH} 7.5,4 \mathrm{~m} M \mathrm{MgCl}_{2}, 25 \mathrm{~m} M \mathrm{NaF}, 2 \mathrm{~m} M \beta-$ mercaptoethanol, $0.1 \mathrm{~m} M$ dUTP and $0.05 \mu \mathrm{Ci} / \mathrm{ml}\left[5-{ }^{3} \mathrm{H}\right] \mathrm{dUTP}$. Reaction mixtures were incubated at $37{ }^{\circ} \mathrm{C}$ for $0-15 \mathrm{~min}$, then boiled for $1 \mathrm{~min}$ to stop the reaction. Samples (10 $\mu$ leach) were run out on a thin-layer chromatography sheet (Polygram Cel 300 PEI $\mathrm{UV}_{254}$; Alltech, Deerfield, Ill.) previously spotted with $10 \mathrm{mM}$ 
deoxyuridine nucleotide mixture for visualizing dUMP, dUDP and dUTP. Plates were developed with $0.5 \mathrm{M} \mathrm{LiCl}$ in $2 N$ acetic acid. Following separation, the amount of radioactivity in each deoxyuridine nucleotide fraction was determined by scintillation counting. Enzyme activity was calculated by determining the fraction of radioactivity from each sample in the dUMP form. Total pilomoles of dUMP formed were plotted versus time of incubation and the slope of the resulting line used to determine a rate of enzyme activity in picomoles formed per minute per microgram protein.

Clonogenic assays

Cells were trypsinized and plated at a density of $20000 \mathrm{cells} / \mathrm{cm}^{2}$. After $48 \mathrm{~h}$, medium supplemented with dialyzed fetal bovine serum (Gibco) and the appropriate drug concentration was added to the cells. (Cells were treated with at least ten times the $\mathrm{IC}_{50}$ for growth inhibition as determined after a 48-h drug treatment.) Drug medium on 48 -h samples was replenished after $24 \mathrm{~h}$. At 24 or $48 \mathrm{~h}$, cells were trypsinized and replated in $10 \mu M$ thymidine medium (except $\mathrm{CdA}$ experiments) to restore thymidine levels. After a further 12 days, surviving colonies were fixed with a 3:1 methanol/ acetic acid solution and stained with trypan blue. Surviving fractions represent the plating efficiency for a given timepoint divided by the control plating efficiency. Control plating efficiencies were 0.6-0.8 for dutE7 cells, $0.3-0.5$ for con3 cells and 0.4-0.6 for HuTu80-derived clones.

DNA fragmentation analysis

with pulsed field gel electrophoresis (PFGE)

Cells were plated as above with the addition of a 24-h labeling period with $0.1 \mu \mathrm{Ci} / \mathrm{ml}\left[2-{ }^{14} \mathrm{C}\right]$ thymidine $[0.15 \mu \mathrm{Ci} / \mathrm{ml} ; 56 \mathrm{mCi} /$ mmol; Moravek Biochemicals, Brea, Calif.] and a 16-h chase period prior to drug treatment. Cells were resuspended at $10^{7}$ cells $/ \mathrm{ml}$ as described previously [3] in a final concentration of $0.7 \%$ low melting point agarose (BRL). Cell blocks containing $2-3 \times 10^{5}$ cells were loaded onto a $0.7 \%$ agarose gel (Bio-Rad) and analyzed with PFGE using a CHEF DR-II apparatus. Samples were run in $14{ }^{\circ} \mathrm{C}$ recirculating $0.5 \times$ TBE buffer $(45 \mathrm{~m} M$ Tris borate/ $1 \mathrm{~m} M$ EDTA, $\mathrm{pH} 8.0)$ at $1.9 \mathrm{~V} / \mathrm{cm}$ with a reorientation angle of $120^{\circ}$. The switching interval was ramped linearly from 1200 to $2400 \mathrm{~s}$ over $48 \mathrm{~h}$. Lanes were cut into 8-mm slices, melted in $0.1 \mathrm{~N} \mathrm{HCl}$ and analyzed by scintillation counting. $F_{\text {released }}$ values represent the fraction of total counts per minute which migrated from the cell block into the gel. Control $F_{\text {released }}$ values varied from 0.02 to 0.07 for all cell lines.

Quantification of single strand DNA breaks (SSB)

in FdUrd-treated cells with alkaline elution

Con 3 and dutE7 cells were plated and labeled with $0.03 \mu \mathrm{Ci} / \mathrm{ml}$ $\left[2-{ }^{14} \mathrm{C}\right]$ thymidine for $48 \mathrm{~h}$ prior to incubation with $100 \mathrm{n} M$ FdUrd for 10,16 or $24 \mathrm{~h}$. Control samples were chased for $10 \mathrm{~h}$. Alkaline elution was performed as described previously [4, 15]. Cells were collected in $15 \mathrm{~m} M$ EDTA/PBS, and approximately 300000 cells were loaded onto a Nucleopore polycarbonate filter (pore size $2 \mu \mathrm{m}$; Costar), washed with PBS and lysed in a solution of $2 \%$ sodium dodecyl sulfate (SDS), $0.05 M$ glycine and $0.02 M \mathrm{Na}_{2-}$ EDTA, pH 9.6. Cells were further digested with $0.5 \mathrm{mg} / \mathrm{ml}$ proteinase $\mathrm{K}$ in the described lysis buffer for $30 \mathrm{~min}$. DNA was then eluted by perfusing the filter with tetrapropylammonium hydroxide solution, $\mathrm{pH} 12.2$, containing $0.02 M$ EDTA and $0.1 \%$ SDS, at a rate of $2 \mathrm{ml} / \mathrm{h}$ for $10 \mathrm{~h}$. Eluted material was collected in fractions at 2 -h intervals. The radioactivity in each fraction and that remaining on the filter were measured with scintillation counting. Cells irradiated on ice with 1.5 Gy were analyzed as external standards as described previously [4]. Data are expressed as the percent of ra-
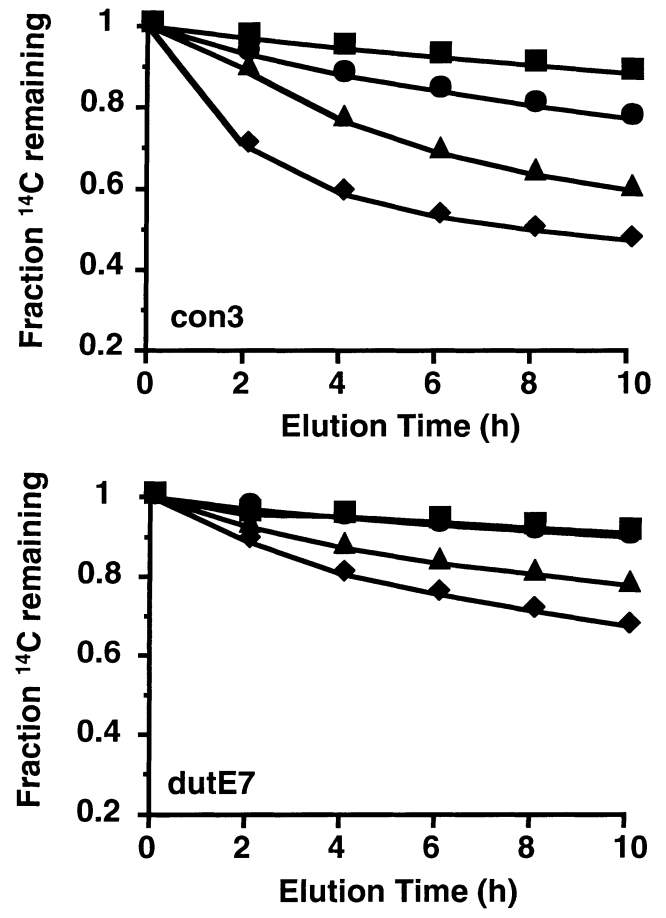

Fig. 2A,B Alkaline elution of parental DNA following treatment with $100 \mathrm{n} M$ FdUrd. Con3 cells (A) and dutE7 cells (B) were prelabelled with $\left[{ }^{14} \mathrm{C}\right]$-thymidine and treated with $100 \mathrm{n} M$ FdUrd for $0(\boldsymbol{\nabla}), 10(\boldsymbol{\bullet}), 16(\boldsymbol{\Delta})$ or $24 \mathrm{~h}(\bullet)$ prior to analysis by alkaline elution. At each timepoint, more DNA eluted from con3 than from dutE7 cell lysates. The plots shown are representative of four separate experiments

dioactivity in a given fraction relative to the total amount of radioactivity in the sample.

\section{Results}

Effect of dUTPase expression on FdUrd-induced single-strand break formation

Previous PFGE studies have revealed that dutE expression can partially protect dutE7 cells from FdUrdinduced DNA double strand breaks (DSB) [5]. We used alkaline elution to examine DNA from FdUrd-treated con3 and dutE7 cells and thereby determine whether SSB formation in parental DNA strands was inhibited in dutE7 cells as well. Figure 2A,B contain data from a representative elution experiment of DNA from con3 and dutE7 cells treated with $100 \mathrm{n} M$ FdUrd for 10,16 or $24 \mathrm{~h}$. Although there was no difference in basal SSBs between the two cell lines, after either 16 or $24 \mathrm{~h}$ of FdUrd treatment there was significantly less damage in dutE7 than con3 cell DNA. To examine the kinetics of SSB formation in both cell lines, values for the third fraction from several experiments were combined and plotted (Fig. 3) as previously described [4]. Parental strand SSB accumulation measured in con3 DNA at 10 or $16 \mathrm{~h}$ was comparable to that measured in dutE7 DNA at 16 or $24 \mathrm{~h}$, respectively. 


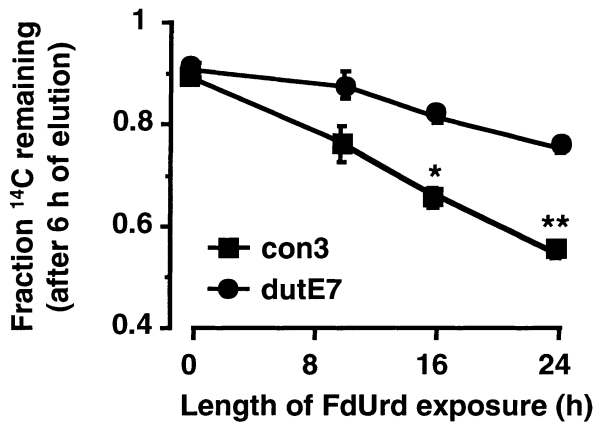

Fig. 3 Summary of multiple alkaline elution experiments showing time dependence of SSB accumulation in con3 and dutE7 cell parental DNA. Values for the fraction of ${ }^{14} \mathrm{C}$ remaining on the filter after $6 \mathrm{~h}$ of elution were averaged to establish a timecourse for FdUrd-induced SSB in con3 and dutE7 cells. DutE7 cells were significantly less sensitive to FdUrd-induced SSB accumulation at the 16 and 24 h timepoints $(P<0.05$ and $P<0.001$, respectively, Student's $t$-test). Data are expressed as the means \pm standard error $(n=4)$

Pharmacological specificity of dUTPase-mediated protection from drug treatments

To evaluate the pharmacological specificity of the protective effects of dutE expression, we treated dutE7 and con3 cells with two other known TS inhibitors, CB3717 and MTX. After $24 \mathrm{~h}$ treatment with $100 \mu M$ CB3717, dutE7 cells were threefold less sensitive to CB3717-induced loss of clonogenicity, and twofold less sensitive to CB3717-induced DNA fragmentation (Figs. 4A and 5A). Similarly, dutE7 cells treated with $500 \mathrm{n} M$ MTX were 1.5-fold less sensitive than con3 cells to MTX-induced cytotoxicity (Fig. 4B). There was no significant MTX-induced DNA fragmentation at $24 \mathrm{~h}$ (Fig. 5B). After a 48-h exposure to CB3717, dutE7 and con3 cells were comparable in their loss of clonogenicity, even though dutE7 cells were still twofold less sensitive to CB3717-induced DNA fragmentation (Fig. 5A). Similarly, MTX cytotoxicity was comparable in the two cells lines at $48 \mathrm{~h}$ when dutE7 cells were threefold less sensitive to MTX-induced DNA fragmentation. MTX-induced DNA damage and cytotoxicity were reversible with $10 \mu M$ thymidine at both 24 and $48 \mathrm{~h}$ (data not shown). DutE7 and con3 cells were equally sensitive to $6 \mu M 2$ CdA, a DNA synthesis inhibitor whose actions are independent of dTTP or dUTP levels (Figs. 4C and 5C).

Cell line specificity of dUTPase-mediated protection from FdUrd treatment

We also examined the effects of dutE expression in a second gastrointestinal tumor cell line, HuTu80. Following retroviral infection with pLNCX.dutE, two clones were isolated, $80 \mathrm{C}$ and $80 \mathrm{~K}$, which had dUTPase activities of $51.8 \pm 5.6$ and $59.7 \pm 9.0 \mathrm{pmol} /$ $\min$ per $\mu \mathrm{g}$ protein, respectively, compared with $19 \pm 1.6 \mathrm{pmol} / \mathrm{min}$ per $\mu \mathrm{g}$ protein in parental HuTu 80 cells. The dutE-expressing HuTu80 clones were as sen-
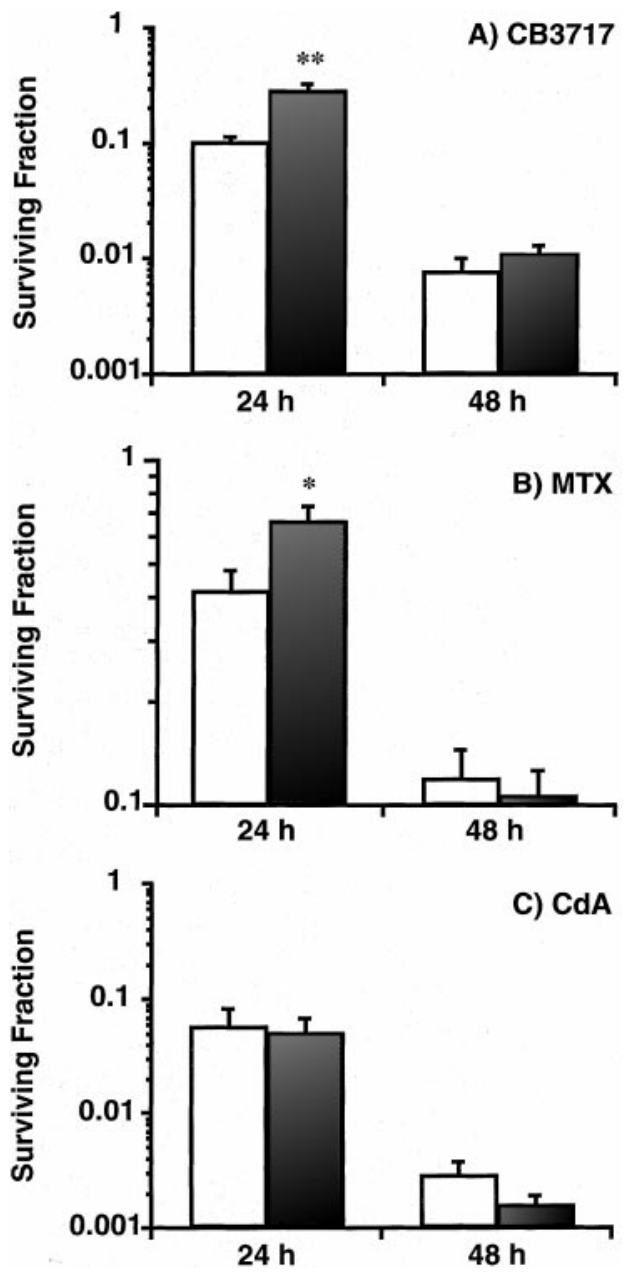

Fig. 4A-C Drug-induced loss of clonogenicity in con3 and dutE7 cells following exposure to CB3717 (A), MTX (B) or CdA (C). Con3 ( $\square$ ) and dutE7 ( $\square$ ) cells were treated with either $100 \mu M$ CB3717, $500 \mathrm{n} M$ MTX or $6 \mu M \mathrm{CdA}$ for 24 or $48 \mathrm{~h}$ and assayed for loss of clonogenicity. After $24 \mathrm{~h}$, dutE7 cells were significantly less sensitive to CB3717-induced cytotoxicity $(P<0.01$, MannWhitney test). DutE7 cells were also less sensitive to MTX-induced cytotoxicity at $24 \mathrm{~h}(P<0.02$, Student's $t$-test $)$. However, by $48 \mathrm{~h}$ of drug treatment, there were no differences in either CB3717- or MTX-induced cytotoxicity between the two cell lines. There was also no difference in CdA sensitivity between con3 and dutE7 cells. Data are expressed as the means \pm standard error $(n \geq 5)$

sitive as the parental line to the cytotoxic and DNAdamaging effects of FdUrd (Fig. 6). There was also no difference in FdUrd-induced SSB formation between HuTu 80 cells and dutE clones $80 \mathrm{C}$ and $80 \mathrm{~K}$ (data not shown).

\section{Discussion}

One question that remained unanswered by our previous work was whether the reduction in FdUrd-induced DSB formation afforded by dUTPase expression was the result of protection from parental strand SSB formation. Figures 2 and 3 indicate that, indeed, the appearance of SSBs in the parental DNA strands of FdUrd-treated 

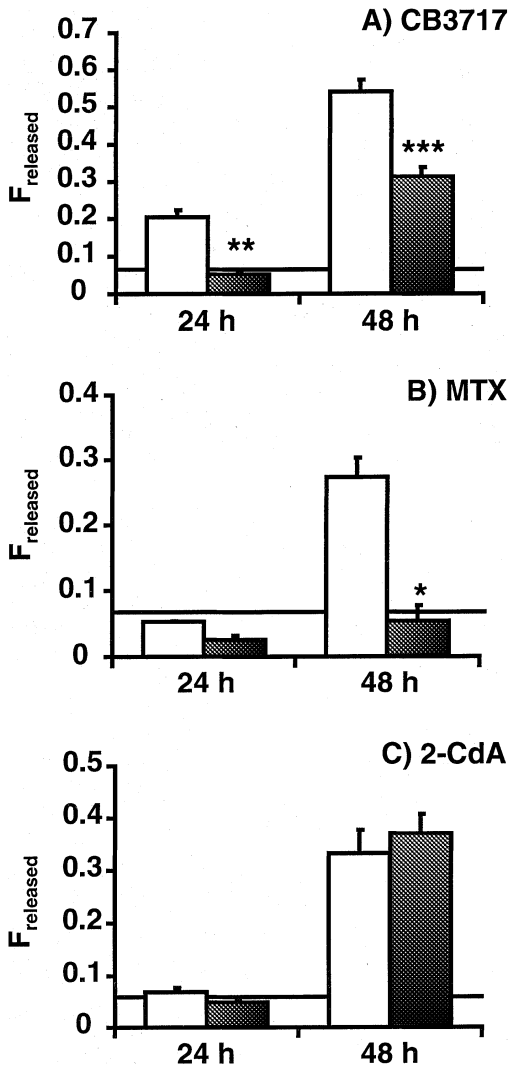

Fig. 5A-C Drug-induced DNA DSB formation in con3 and dutE7 cells as measured by PFGE. Con3 ( $\square$ ) and dutE7 (ם) cells were treated as described in Fig. 4 legend and assayed for DNA DSB formation with PFGE. Data are expressed as the means \pm standard error $(n \geq 4)$ of the fraction of DNA released in the gel $\left(\mathrm{F}_{\text {released }}\right)$. DutE7 cells were significantly less sensitive to CB3717induced DNA fragmentation at both the 24 and $48 \mathrm{~h}$ timepoints $(P$ $<0.0001$ and $P<00005$, respectively, Student's $t$-test). DutE7 cells were also less sensitive to MTX-induced DNA DSB at the $48 \mathrm{~h}$ timepoint $(P<0.005)$. The solid line represents background DNA fragmentation for both cell lines

dutE7 cells was delayed by about $6-8 \mathrm{~h}$, compared with their time course of appearance in con 3 cells. This result, and the observation that the appearance of FdUrd-induced SSBs precedes DSB formation, reinforce the model that FdUrd-induced DSBs arise from processing of previously created parental strand SSBs, in some manner which has yet to be determined. However, the observation that dutE expression inhibits SSB formation argues against another previously proposed model which suggests that parental SSBs arise from inefficient or abortive repair of uracil that appears in DNA as a result of spontaneous deamination of cytosine residues in the parental strand $[6,16]$. If this second model were correct, there would be no difference in SSB formation between con3 and dutE7 cells as dUTPase would not affect excision of uracil from the parental DNA strand. We recently proposed an alternative model in which parental strand SSB result from the attempted mismatch repair of $\mathrm{dA} / \mathrm{dU}$ base pairs following incorporation of dUTP into the nascent strand [20]. The ability of dutE to
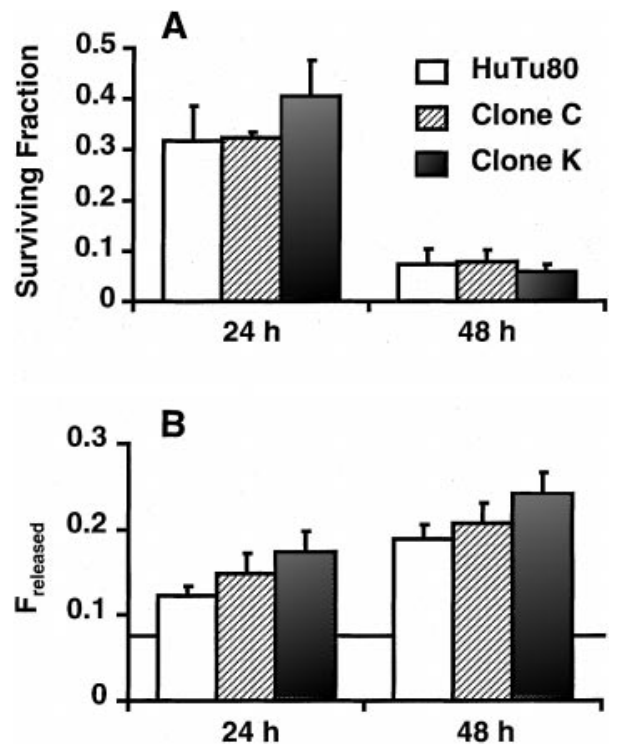

Fig. 6A,B FdUrd-induced loss of clonogenicity and DNA DSB formation in HuTu80 cells. HuTu80 clones were treated with $100 \mathrm{n} M$ FdUrd for 24 or $48 \mathrm{~h}$ and assayed for loss of clonogenicity (A) or DNA DSB formation with PFGE (B). No differences in FdUrd sensitivity were observed between the parental HuTu80 cells and E. coli dUTPase-expressing clones $80 \mathrm{C}$ and $80 \mathrm{~K}$. Data are expressed as the means \pm standard error $(n \geq 3)$

inhibit SSB formation in HT29 cells is consistent with this model.

Another question that remained unanswered in our previous study was whether the protection from FdUrdinduced DNA damage and cytotoxicity was in part due to reduced incorporation of FdUTP into DNA. We addressed this question indirectly by testing the sensitivities of con3 and dutE7 cells to other TS inhibitors. DNA fragmentation and loss of clonogenicity were both diminished in dutE7 cells treated with either CB3717 (which inhibits TS directly) or MTX (which inhibits TS indirectly, by depleting reduced folate cofactors). This result supports the hypothesis that the protective effects of dutE expression in HT29 cells are related to TS inhibition in general, and not to FdUrd treatment specifically. As was the case with FdUrd, protection from cytotoxicity was dependent on the length of drug exposure for both of these drugs, with statistically significant protection observed after $24 \mathrm{~h}$ drug treatment but not after $48 \mathrm{~h}$ treatment (Fig. 4). To confirm that the protective effects of dUTPase expression in this cell line are indeed due to its antagonism of the consequences of TS inhibition, and not to some less specific effect on repair of DNA damage, we challenged con3 and dutE7 cells with CdA, which has no known effect on TS (Fig. 4C). Neither CdA-induced DNA fragmentation nor cytotoxicity were prevented by dUTPase expression in this cell line. These results further support the expected specificity of dUTPase protection against agents whose DNA damaging effects occur via TS inhibition.

The data also suggest that uracil misincorporation/ misrepair may contribute more to drug-induced DNA 
damage than cytotoxicity, in HT29 cells. DutE7 cells were significantly protected from DNA damage under conditions where they were equally sensitive to druginduced cytotoxicity (Fig. 5). This result further suggests that the observed cytotoxicity was not entirely dependent upon DNA DSB formation. After treatment with MTX for $48 \mathrm{~h}$, dutE7 cells suffered a $90 \%$ loss in clonogenicity with no apparent DNA DSB formation (Figs. 5B and 6B). This cytotoxicity did not appear to result from purine depletion as it was completely reversible with $10 \mu M$ thymidine (data not shown).

Finally, to test the cell line specificity of dUTPase protection from the effects of TS inhibition, we derived clones from another gastrointestinal tumor cell line, HuTu80, whose response to FdUrd we and others have previously documented $[3,22]$. In contrast to the case in the HT29 clones, dutE expression conferred no protection from FdUrd in HuTu80 cells (Fig. 6). This observation is supported by work from other laboratories which suggests that the contributions of uracil misincorporation/misrepair to FdUrd cytotoxicity vary between cells lines [1]. The inability of dutE to protect HuTu80 cells could result from several factors. HuTu80 cells have a relatively high level of endogenous dUTPase activity which could already be sufficient for suppressing dUTP levels. Alternatively, HuTu80 cells may be more sensitive to DNA synthesis inhibition resulting from dTTP depletion, than uracil misincorporation/misrepair.

The results from this study suggest that the role of uracil misincorporation/misrepair in cytotoxicity induced by TS inhibition is both time- and cell line-dependent. It is unclear why the protective effects of dutE expression in HT29 cells were observed after $24 \mathrm{~h}$, but not after $48 \mathrm{~h}$, of drug treatment. The variability in the importance of uracil misincorporation in responses of different cell lines to TS inhibition, however, is not unexpected. A cell's sensitivity to TS inhibition and dTTP depletion or DNA synthesis inhibition, its level of uracil DNA glycosylase activity and its ability to initiate programmed cell death processes are all factors that could contribute to this variability. We are currently investigating the role of this third factor, uracil DNA glycosylase, in FdUrd-induced cytotoxicity and DNA damage in HT29 cells.

Acknowledgements We would like to thank Dr Ann Jackman for providing CB3717, Dr Jack Dixon for providing pGEXKT and BL21 E. coli and Dr Kevin McDonagh from providing the $\mathrm{GP}+\mathrm{E} 86$ and GP + envAM12 packaging cell lines. We would also like to thank Dr Mary Davis for instruction in alkaline elution and Dr Carolyn Minth for her advice on the pGEX/BL21 system.

\section{References}

1. Brown SD, Ladner R, Maybaum J, Aherne GW (1997) Deoxyuridine triphosphate (dUTP) accumulation: relevance to the cytotoxic effects of thymidylate synthase (TS) inhibitors (Abstract). Proc Am Assoc Cancer Res 38: 477

2. Brynoff K, Eliasson R, Reichard P (1978) Formation of Okazaki fragments in polyoma DNA synthesis caused by misincorporation of uracil. Cell 13: 573
3. Canman CE, Tang H-Y, Normolle DP, Lawrence TS, Maybaum J (1992) Variations in patterns of DNA damage induced in human colorectal tumor cells by 5-fluorodeoxyuridine implications for mechanisms of resistance and cytotoxicity. Proc Natl Acad Sci USA 89: 10474

4. Canman CE, Lawrence TS, Shewach DS, Tang H-Y, Maybaum J (1993) Resistance to fluorodeoxyuridine-induced DNA damage and cytotoxicity correlates with an elevation of dUTPase activity and failure to accumulate dUTP. Cancer Res 53: 5219

5. Canman CE, Radany EH, Parsels LA, Davis MA, Lawrence TS, Maybaum J (1994) Induction of resistance to fluorodeoxyuridine cytotoxicity and DNA damage in human tumor cells by expression of Escherichia coli deoxyuridinetriphosphatase. Cancer Res 54: 2296

6. Curtin NJ, Harris AL, Aherne GW (1991) Mechanism of cell death following thymidylate synthase inhibition: 2 '-deoxyuridine-5'-triphosphate accumulation, DNA damage, and growth inhibition following exposure to CB3717 and dipyridamole. Cancer Res 51: 2346

7. el-Hajj HH, Zhang H, Weiss B (1988) Lethality of a dut (deoxyuridine triphosphatase) mutation in Escherichia coli. J Bacteriol 170: 1069

8. Goulian M, Bleile B, Tseng BY (1980) The effect of methotrexate on levels of dUTP in animal cells. J Biol Chem 255: 10630

9. Goulian M, Bleile B, Tseng BY (1980) Methotrexate-induced misincorporation of uracil into DNA. Proc Natl Acad Sci USA 77: 1956

10. Grafstrom RH, Tseng BY, Goulian M (1978) The incorporation of uracil into animal cell DNA in vitro. Cell 15: 131

11. Guan KL, Dixon JE (1991) Eukaryotic proteins expressed in Escherichia coli: an improved thrombin cleavage and purification procedure of fusion proteins with glutathione S-transferase. Anal Biochem 192: 262

12. Hakes DJ, Dixon JE (1992) New vectors for high level expression of recombinant proteins in bacteria. Anal Biochem 202: 293

13. Heidelberger C, Danenberg PV, Moran RG (1983) Fluorinated pyrimidines and their nucleosides. Adv Enzymol Relat Areas Mol Biol 54: 58

14. Ingraham HA, Dickey, L Goulian M (1986) DNA fragmentation and cytotoxicity from increased cellular deoxyuridylate. Biochemistry 25: 3225

15. Kohn KW, Ewig AG, Erickson LC, Zwelling LA (1981) DNA repair, a laboratory manual of research procedures. Marcel Dekker, New York, p. 379

16. Li JC, Kaminskas E (1984) Accumulation of DNA strand breaks and methotrexate cytotoxicity. Proc Natl Acad Sci USA 81: 5694

17. Lindahl T (1979) DNA glycosylases, endonucleases for apurinic/apyrimidinic sites, and base excision-repair. Prog Nucl Acid Res Mol Biol 22: 135

18. Markowitz D, Goff S, Bank A (1988) A safe pacakaging line for gene transfer: separating viral genes on two different plasmids. J Virol 62: 1120

19. Markowitz D, Goff S, Bank A (1988) Construction and use of a safe and efficient amphotropic packaging cell line. Virology 167: 400

20. Parsels LA, Zellars RC, Loney TL, Parsels JD, Clarke MF, Lawrence TS, Maybaum J (1997) Prevention of fluorodeoxyuridine-induced cytotoxicity and DNA damage in HT29 colon carcinoma cells by conditional expression of wild-type p53 phenotype. Mol Pharmacol 52: 600

21. Sedwick WD, Kutler M, Brown OE (1981) Antifolate-induced misincorporation of deoxyuridine monophosphate into DNA: inhibition of high molecular weight DNA synthesis in human lymphoblastoid cells. Proc Natl Acad Sci USA 78: 917

22. Washtien WL (1982) Thymidylate synthetase levels as a factor in 5-fluorodeoxyuridine and methotrexate cytotoxicity in gastrointestinal tumor cells. Mol Pharmacol 21: 723 